\title{
Functional microdomains in bacterial membranes
}

\author{
Daniel López ${ }^{1}$ and Roberto Kolter ${ }^{2}$ \\ Department of Microbiology and Molecular Genetics, Harvard Medical School, Boston, Massachusetts 02115, USA
}

\begin{abstract}
The membranes of eukaryotic cells harbor microdomains known as lipid rafts that contain a variety of signaling and transport proteins. Here we show that bacterial membranes contain microdomains functionally similar to those of eukaryotic cells. These membrane microdomains from diverse bacteria harbor homologs of Flotillin-1, a eukaryotic protein found exclusively in lipid rafts, along with proteins involved in signaling and transport. Inhibition of lipid raft formation through the action of zaragozic acid-a known inhibitor of squalene synthases-impaired biofilm formation and protein secretion but not cell viability. The orchestration of physiological processes in microdomains may be a more widespread feature of membranes than previously appreciated.
\end{abstract}

[Keywords: Lipid rafts; biofilm formation; Flotillin-1; bacteria; Bacillus subtilis; Staphylococcus aureus]

Supplemental material is available at http://www.genesdev.org.

Received May 5, 2010; revised version accepted July 16, 2010.

A feature common to all living cells is the presence of a lipid membrane that defines the boundary between the inside and the outside of the cell. Proteins that localize to the membrane serve a number of essential functions. In eukaryotic cells, membrane proteins that mediate signal transduction and protein secretion are often localized in membrane microdomains enriched in certain lipids, such as sterols and sphingolipids. These microdomains are commonly referred to as "lipid rafts" or "membrane rafts" (Pike 2006; Lingwood and Simons 2010).

The function of proteins associated with lipid rafts depends on the integrity of these areas. Alterations in the composition of lipid rafts lead to defects in cell-cell signaling processes and transduction pathways in which these proteins are involved. Thus, disruptions of lipid rafts are associated with a large variety of human diseases, including Alzheimer's, Parkinson's, cardiovascular, and prion diseases (Michel and Bakovic 2007). Because of their profound importance on cell physiology, these membrane domains are interesting targets for the development of new pharmacological approaches to cure and prevent these diseases.

Until now, lipid rafts have been identified and characterized in eukaryotic cells. However, many bacterial membrane proteins involved in cell-cell signaling and

${ }^{1}$ Present address: Institute of Molecular Infection Biology, Würzburg Universität, Josef-Schneider-Straße 2/Bau D15, 97080 Würzburg, Germany.

${ }^{2}$ Corresponding author.

E-MAIL rkolter@hms.harvard.edu; FAX (617) 738-7664.

Article published online ahead of print. Article and publication date are online at http://www.genesdev.org/cgi/doi/10.1101/gad.1945010. signal transduction pathways are distributed heterogeneously across the cytoplasmic membrane (Meile et al. 2006). These observations suggest that specialized membrane microdomains are also a feature of bacterial cells.

Certain proteins of eukaryotic membranes have been consistently described as being present in lipid rafts (Lingwood and Simons 2010). Most prominent among these is the protein alternatively referred to as Flotillin-1, or Reggie, which appears to be involved in the orchestration of diverse processes related to signal transduction, vesicle trafficking, and cytoskeleton rearrangement (Langhorst et al. 2005). Interestingly, bioinformatic analyses indicate that most bacterial genomes encode proteins whose amino acid sequences show some similarity to Flotillin-1. While these bacterial proteins have not been extensively investigated, two reports (Zhang et al. 2005; Donovan and Bramkamp 2009) demonstrated that the flotillin-like proteins from the spore-forming Bacillus halodurans and Bacillus subtilis are distributed heterogeneously in the cytoplasmic membrane displaying a punctate pattern along the entire cell. While no exact function was ascribed to these bacterial proteins, a $B$. subtilis mutant lacking the flotillin-like protein showed a delay in the onset of sporulation and reduced sporulation efficiency (Donovan and Bramkamp 2009). Attempts to determine the type of lipid that led to the punctate distribution of the $B$. subtilis flotillin-like protein were inconclusive, finding only that its localization was not dependent on lipids containing phosphatidylglycerol or cardiolipin (Donovan and Bramkamp 2009). Cardiolipin was of particular interest, because it had been shown to occur in patches in the B. subtilis membrane (Kawai 
et al. 2004; Matsumoto et al. 2006; Mileykovskaya and Dowhan 2009). Thus, the function and lipid association of bacterial flotillin-like proteins remains poorly understood.

All members of the Flotillin family of proteins are members of a superfamily of proteins that contains "SPFH" or "PHB" domains (named after the proteins Stomatin, Prohibitin, Flotillin, and HflK/C) (Tavernarakis et al. 1999; Browman et al. 2007). SPFH domain-containing proteins are found associated with lipid rafts, and are thought to function in many ways, such as in raft formation, kinase activity enhancement, and ion channel regulation (Morrow and Parton 2005; Kato et al. 2006; Browman et al. 2007). Aside from those bacterial proteins with high sequence similarity to Flotillin-1 presented above, bacteria also encode other SPFH proteins. While these proteins are widely distributed in bacteria, their functions remain poorly understood. The few genetic studies carried out on SPFH proteins have not yielded clear phenotypes; however, they appear to be involved in stress responses such as high-salt and antibiotic treatment (Butcher and Helmann 2006). Importantly, most bacterial genomes encode multiple SPFH proteins, and thus some of their function may be redundant.

Here we present evidence that bacteria contain lipid rafts that are functionally similar to those found in eukaryotes, in that they harbor and organize proteins involved in signal transduction, small molecule translocation, and protein secretion. The lipids associated with the bacterial rafts are probably polyisoprenoids synthesized via pathways that involve squalene synthases; inhibitors of this enzyme interfere with the formation of bacterial lipid rafts. In addition, we demonstrate a function for the lipid rafts: A mutant devoid of SPFH proteins is defective in a signal transduction pathway whose sensor kinase is found in the rafts. All of these results are consistent with the idea that the orchestration of physiological processes in microdomains may be a more widespread feature of membranes than previously appreciated.

\section{Results}

\section{A lipid synthesis gene involved in signaling biofilm formation in B. subtilis}

We discovered the existence of bacterial lipid rafts while conducting analyses of the signaling pathway involved in biofilm formation by $B$. subtilis. Recently, we reported that the antifungal agent nystatin and the $B$. subtilis molecule surfactin can signal this bacterium to induce biofilm formation by activating a signal transduction pathway whereby the membrane histidine kinase KinC phosphorylates the transcription factor Spo0A (Fig. 1A; Lopez et al. 2009). The fact that nystatin had an observable effect on $B$. subtilis was surprising, because the drug specifically inhibits fungal growth. This inhibition is accomplished through nystatin's ability to bind to, and displace from the membrane, the fungal sterol ergosterol (Bolard 1986). Importantly, ergosterol is not synthesized by bacteria. This led us to hypothesize that, in B. subtilis,
A

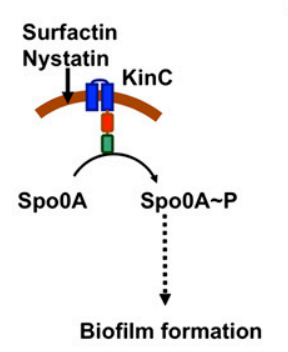

B

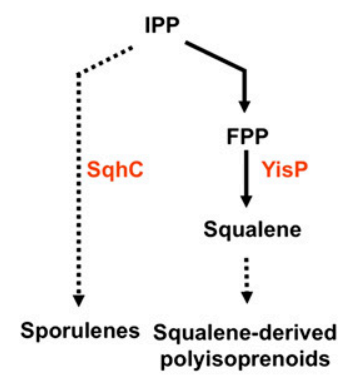

C

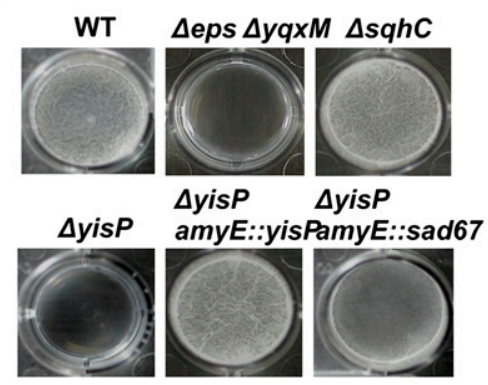

Figure 1. yis $P$ affects the pathway to biofilm formation. $(A)$ Schematic representation of the signaling pathway leading to biofilm formation in $B$. subtilis. The pathway is triggered by activation of the master regulator Spo0A via phosphorylation by KinC. Dashed lines represent indirect activation. $(B)$ Putative metabolic pathway to the formation of distinct polysioprenoids in B. subtilis. Enzymes discussed in the text are written in red, next to the reaction they catalyze. Dashed lines represent unknown steps. (C) Pellicle formation assay to test biofilm formation in different strains of $B$. subtilis. Positive and negative controls are represented by the wild-type strain (WT) and the matrix-deficient mutant ( $\Delta$ eps $\Delta y q x M$ ) (Branda et al. 2004, 2006).

there might exist a membrane molecule analogous to ergosterol involved in the signal transduction pathway leading to biofilm formation.

While $B$. subtilis membranes do not contain sterols, structurally similar molecules termed sporulenes have been described recently in this bacterium (Fig. 1B; Bosak et al. 2008; Kontnik et al. 2008). Both ergosterol and sporulenes are synthesized from the common precursor isoprenyl pyrophosphate (IPP). However, while ergosterol is derived from squalene, this is not the case for sporulenes. Instead, sporulenes are synthesized through a pathway involving the product of the $s q h C$ gene, a putative polyisoprenoid cyclase that remains uncharacterized (Bosak et al. 2008; Kontnik et al. 2008). To test if sporulenes might be involved in biofilm formation, we deleted the $s q h C$ gene. $B$. subtilis forms floating biofilms (pellicles) when cultures are left standing undisturbed (see Fig. 1C, top left panel). The cells are held together in the pellicle by an extracellular matrix. This matrix is composed of an exopolysaccharide produced by the products of the eps operon and amyloid-like fibers of the protein TasA, whose formation requires the three-gene operon yqxM-sipW-tasA (Branda et al. 2004, 2006; Romero et al. 2010). Thus, a double mutant lacking both operons ( $\Delta$ eps $\triangle y q x M)$ cannot make the matrix and does not form pellicles (see Fig. 1C, top center panel). The $\Delta s q h C$ mutant 
formed pellicles that were indistinguishable from those formed by the wild type, indicating that sporulenes were not involved in the biofilm formation signaling pathway (Fig. 1C, top right panel).

Having ruled out $s q h C$ involvement in biofilm formation, we initiated a search for genes whose products might synthesize molecules related to squalene (Fig. 1B). We carried out a bioinformatic analysis of the B. subtilis genome to identify known or putative genes that might be involved in the pathway(s) from IPP to squalene. From the set of genes thus identified, we focused our efforts on the first nonessential gene found in this putative pathway, yis $P$, whose gene product is predicted to have sequence similarity with phytoene or squalene synthases (Kobayashi et al. 2003). As we show below, the product of yisP is indeed capable of generating a C30 polyisoprenoid. Deletion of yisP resulted in a complete loss of pellicleforming ability (Fig. 1C, bottom left panel). Pellicle formation was restored in the $\triangle y i s P$ mutant by reintroducing a functional copy of the gene into the neutral amyE locus of the chromosome (Fig. 1C, bottom center panel).

To determine where yis $P$ acted in the signaling pathway of biofilm formation, we carried out a series of epistasis experiments. As discussed above, biofilm formation requires phosphorylation of SpoOA (see Fig. 1A). To know whether the action of yis $P$ was before or after the phosphorylation of SpoOA, we introduced a constitutively active allele of $s p o 0 A$ whose product does not require phosphorylation (termed sad67) into the $\Delta y i s P$ mutant (Ireton et al. 1993). This strain formed pellicles, thus indicating that the product of yis $P$ acts upstream of Spo0A phosphorylation and most likely is involved in the function of KinC (Fig. 1C, bottom right panel). Because of the pleiotropic effects of expressing the sad67 allele, the formation of pellicles was not as robust as in the wild type. However, the action of yis $P$ upstream of matrix gene expression was confirmed by double-mutant analyses, where we deleted two repressors of matrix synthesis genes and obtained robust pellicles (Supplemental Fig. S1). Based on these results, we proceeded to test predictions of the hypothesis that YisP was important for the function of KinC. We used a chimeric construct of the kinase kinC that had replaced the phosphorylation domain of KinC for the phosphorylation domain of the kinase DegS. DegS is the kinase responsible for the inhibition of imotility; thus, addition of the signaling molecule nystatin to agar medium prevented motility in the strain harboring the chimera KinC-DegS (Lopez et al. 2009). When we deleted the gene yisP in the strain harboring the chimera KinC-DegS, the response of the chimeric kinase (inhibition of motility) was not observed when nystatin was present (Supplemental Fig. S2). This result confirmed our hypothesis that the product of the gene yisP is important for the function of the kinase KinC

The product of yisP displays squalene synthase activity in vitro

Since yis $P$ was a gene of unknown function, we decided to characterize the activity of its product to gain some insights into how it might be involved upstream of Spo0A phosphorylation. We cloned and expressed the yisP gene in Escherichia coli. As expected from its predicted amino acid sequence, purified recombinant YisP had the enzymatic features of a phytoene or squalene synthase, and preferentially used farnesyl pyrophosphate (FPP) as a substrate (Supplemental Fig. S3; Lee and Poulter 2008). In addition, YisP enzymatic activity was dependent on $\mathrm{NADH}$ and was blocked by nanomolar concentrations of the competitive inhibitor of squalene synthases, zaragozic acid (Fig. 2A; Bergstrom et al. 1993). While squalene itself is not a colored compound, many molecules derived from squalene are indeed pigments; e.g., carotenoids. In this respect, it was interesting to discover that the yis $P$ mutant lost a dark-orange pigment that is apparent in the wild type (Fig. 2B). As an initial characterization of this change in pigmentation, we carried out preliminary lipid analyses on the membranes of wild type and the $\Delta y i s P$ mutant. We extracted lipids from the pellet of the cultures of the wild-type strain and the $\Delta y i s P$ mutant, and we carried out liquid chromatography/mass spectroscopy (LC-MS) analyses of these. Wild-type extract displayed two adjacent peaks in its profile that were absent in the profile of the $\Delta y i s P$ mutant (Supplemental Fig. S4). These potentially unidentified molecules will require complete characterization, yet this is beyond the scope of this initial report on lipid rafts.

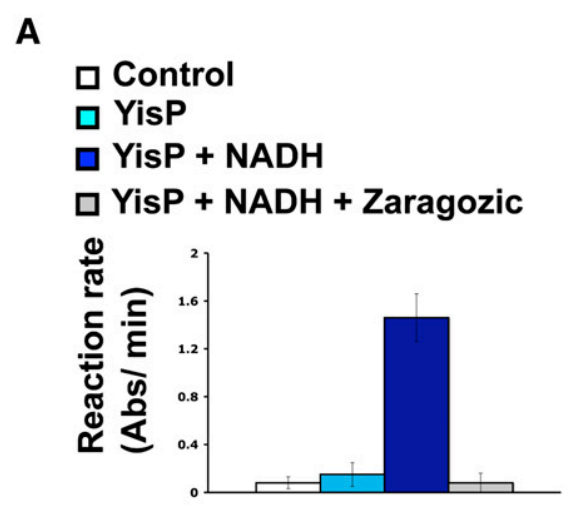

B

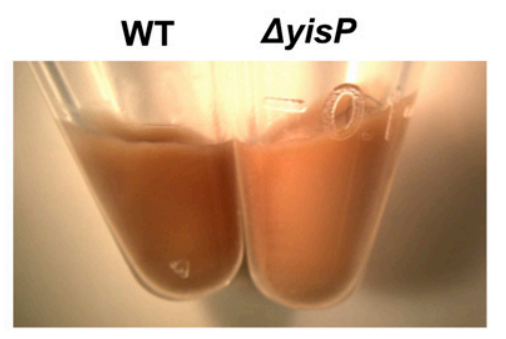

Figure 2. YisP has squalene synthase activity and is involved in the production of a carotenoid. (A) Enzymatic activity of purified YisP from $B$. subtilis under different conditions. FPP at 3.7 $\mu \mathrm{M}$ was used as substrate under the optimal conditions specified in Supplemental Figure S2. Control reaction was performed with no enzyme added. $(B)$ The $\Delta y i s P$ mutant does not produce a dark-orange pigment associated with the cells, in comparison with the wild-type strain. 
The observation that some lipids were absent in the $\Delta y i s P$ mutant led us to test squalene and the two squalenederived molecules $\beta$-carotene and retinol for their ability to restore biofilm formation to the yis $P$ mutant. Biofilm formation was partially restored in the presence of squalene, $\beta$-carotene, and retinol (Supplemental Fig. S5). We thus concluded that a polyisoprenoid lipid, likely to be a $\mathrm{C}_{30}$ triterpenoid, was involved in the signal transduction pathway that activates KinC and results in biofilm formation.

\section{KinC and flotillin colocalize in cytoplasmic membrane puncta}

The finding that a squalene-derived polyisoprenoid was involved in signaling during biofilm formation led us to hypothesize that such a lipid could function to organize KinC in membrane microdomains analogous to lipid rafts in eukaryotic cells. This concept was supported by the finding that not all membrane kinases were affected by the absence of the hypothetical polyisoprenoid $(\Delta y i s P$ mutant). We showed that ComP, a membrane histidine kinase involved in competence development, remained functional in the $\Delta y i s P$ mutant (data not shown). In addition, KinC has been reported to be heterogeneously localized in the membrane of $B$. subtilis (Meile et al. 2006). All of these observations added support to the idea that lipid rafts might exist in $B$. subtilis. Thus, we used standard methods developed to characterize eukaryotic membranes to determine if similar membrane microdomains exist in B. subtilis, and to test if KinC indeed localizes to these microdomains.

Eukaryotic membranes partition into detergent-resistant (DRM) and detergent-sensitive (DSM) fractions. While it is important to emphasize that the DRM fraction is not to be equated with lipid rafts, there is evidence that this fraction includes many of the proteins thought to be present in lipid rafts (Brown 2002). Treatment of eukaryotic cells with Triton X-100 allows for isolation of a DRM fraction, and this can be further fractionated by zonal centrifugation in sucrose gradients. We used this technique to isolate the DRM fraction from $B$. subtilis. The proteins present in the DRM fraction were analyzed by SDS-PAGE in parallel with the proteins present in the DSM fraction (Fig. 3A). For wild-type B. subtilis, the protein profiles from DRM and DSM were dramatically different, suggesting a heterogeneous distribution of lipids and proteins in B. subtilis membranes. Importantly, the number and intensity of protein bands in the DRM was greatly decreased in the $\triangle y i s P$ mutant, or when wildtype cells were treated with zaragozic acid. The DSM fraction of the $\Delta y i s P$ mutant still contains significant amounts of protein. However, the protein profile per se displayed some changes, probably as a consequence of the pleiotropic effect caused by the mutation in $\Delta y i s P$ itself (Supplemental Fig. S6).

In order to detect KinC in either the DSM or DRM fractions, we complemented the $\Delta$ kin $C$ mutant with the translational fusion KinC-YFP (yellow fluorescent protein), and detected the protein by immunoblotting using
A

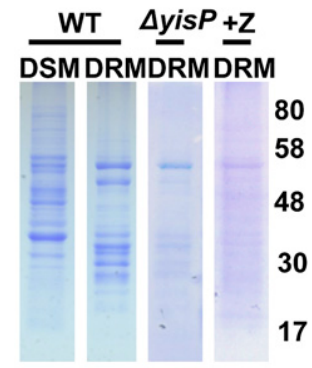

B

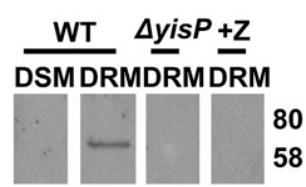

Figure 3. Membrane fractionation and detection of KinC. $(A)$ Membrane fractionation according to differential sensitivity to detergent solubilization. The membrane fractions sensitive and resistant to detergent solubilization are named DSM and DRM, respectively. Membrane proteins associated with each fraction are shown in an SDS-PAGE. DRM-associated proteins decreased in the $\triangle y i s P$ mutant and in the wild-type strain treated with zaragozic acid (+Z lane). (B) Immunoblot analysis detecting KinC in each fraction. Numbers represent molecular weight standards in kilodaltons.

monoclonal antibodies against YFP. KinC was present only in the DRM fraction, and was not detected in the $\Delta y i s P$ mutant or after treatment with zaragozic acid (Fig. $3 \mathrm{~B}$ ). It is possible that, in the absence of lipid rafts, KinC is degraded more quickly because it fails to properly localize in the membrane.

The presence of a sensor kinase in the bacterial DRM fraction suggested a functional similarity with eukaryotes. In eukaryotes, lipid rafts harbor sensor kinases along with protein complexes involved in molecule trafficking and protein secretion (Allen et al. 2007). We thus set out to identify the most abundant proteins found in the DRM fraction using mass spectrometry. The majority of the proteins we identified were involved in signaling, molecule trafficking, and protein secretion (Supplemental Table S1). For example, we detected proteins required for uptake of signaling peptides and siderophores, antibiotic export, and protease secretion (Kontinen and Sarvas 1993; Lazazzera et al. 1997; Dorenbos et al. 2002; Ollinger et al. 2006). As stated above, the proteins identified in the DRM fraction are not necessarily in lipid rafts. However, some of them could be, and would thus be expected to colocalize.

Interestingly, one of the proteins present in the DRM fraction was YuaG, a B. subtilis flotillin-like protein, corroborating the results of Donovan and Bramkamp (2009). As presented above, in eukaryotic cells, Flotillin-1 is localized exclusively in lipid rafts, and appears to orchestrate diverse processes related to signal transduction, vesicle trafficking, and cytoskeleton rearrangement (Langhorst et al. 2005; Morrow and Parton 2005). YuaG shares $39 \%$ amino acid sequence identity (69\% similarity) 
with Flotillin-1. Because of the sequence and localization similarity of YuaG to Flotillin-1, we renamed it FloT. We visualized the membrane distribution of FloT by constructing a translational fusion with YFP. Fluorescence of FloT-YFP was observed distributed in areas along the cytoplasmic membrane (Fig. 4A), and to be highly dynamic (Fig. 7, below), consistent with a prior report of heterogeneous distribution of YuaG (Donovan and Bramkamp 2009) and different from the homogeneous pattern shown by other membrane proteins (Supplemental Fig. S8). This distribution was observed whether the strain producing the FloT-YFP fusion protein contained the wild-type floT allele or a deletion allele $(\Delta f l o T)$ (Supplemental Fig. S9). On average, we detected approximately six foci of FloT localization per B. subtilis cell (Supplemental Table S2).

If the membrane areas where FloT-YFP localized are indeed analogous to eukaryotic lipid rafts, then KinC, present in the DRM fraction, should colocalize with FloTYFP. By expressing the fusion proteins KinC-CFP (Fig. 4B, false-colored in green) and FloT-YFP (Fig. 4B, false-colored in red) simultaneously in a single strain, we were able to test the idea of colocalization of these two proteins. Colocalization of the proteins was indeed detected as a yellow signal, as shown in Figure 4B.

When cells were treated continuously with zaragozic acid, thus inhibiting the enzymatic activity of YisP, the FloT-YFP translational fusion was rarely detected in the vast majority of the cells. We reasoned that inhibition of YisP compromises the integrity of lipid rafts, and thus the proteins that are normally present in these membrane regions (such as flotillin) mislocalize and possibly get degraded. To gain a better insight into this loss of visible FloT-YFP, we analyzed the effect of zaragozic acid treatment on the distribution of FloT-YFP over time (Fig. 4C). At the start of the experiment $(\mathrm{T}=0 \mathrm{~h})$, the cells showed

A

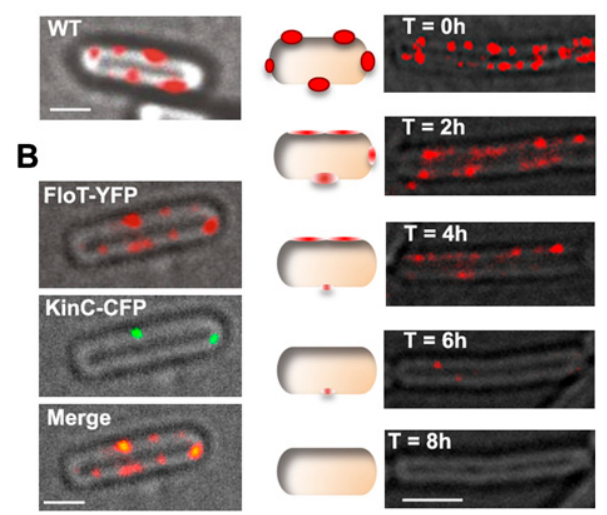

Figure 4. Flotillin and KinC localization in B. subtilis. $(A)$ Cellular localization of FloT-YFP (falsecolored red). (B) Colocalization of FloT and KinC in a double-labeled strain expressing the translational fusions FloT-YFP (false-colored red) and KinCCFP (false colored green). Regions where the two signals overlapped appear yellow in the merge panel. $(C)$ Eight-hour time course following localization of the translational fusion FloTYFP after the addition of zaragozic acid to the sample. Bars: $A, B$, $1 \mu \mathrm{m} ; C, 2 \mu \mathrm{m}$.
FloT-YFP distributed in numerous discrete foci across the membrane. Two hours after zaragozic acid treatment, we observed that these FloT-YFP foci became diffuse throughout the membrane. Diffusion in the localization of FloT-YFP progressed, and the level of the signal diminished over the remainder of the time course. Eventually, by $8 \mathrm{~h}$ after treatment, little or no FloT-YFP signal could be detected. The pattern of other proteins homogeneously distributed across the membrane was not affected when treated with zaragozic acid (Supplemental Fig. S8).

The fact that FloT can localize to discrete foci in membranes containing $\mathrm{C}_{30}$ triterpenoid derived from the enzymatic reaction catalyzed by YisP, coupled to complementation of the lack of YisP with several polyisoprenoids, suggests that there is broad latitude with respect to the structure of the lipids that will assemble lipid rafts in B. subtilis. In addition to this, these regions can be disrupted by the action of the YisP inhibitor zaragozic acid, which causes FloT to diffuse throughout the membrane, similar to a previous reports of when lipid rafts are disrupted in eukaryotic cells (Watanabe et al. 2009).

\section{B. subtilis SPFH domain-containing proteins enhance KinC activity}

The finding that KinC and FloT colocalized led us to ask whether FloT might have some effect on KinC function. When we tested a $\Delta$ floT mutant for KinC activity, the mutant displayed a wild-type phenotype. However, like all flotillin-like proteins, FloT is a member of the superfamily of proteins that contain "SPFH" domains (Tavernarakis et al. 1999; Browman et al. 2007; Hinderhofer et al. 2009). Bioinformatic analysis of the $B$. subtilis genome using the SMART software indicated that this bacterium encodes only one more protein with an SPFH domain, YqfA (Schultz et al. 1998; Letunic et al. 2009). Interestingly, both flotillin-like proteins FloT and YqfA colocalize in discrete foci across the membrane of the bacterium (Fig. 5A). We thus became interested in determining if eliminating both SPFH domain proteins in B. subtilis would have any effect on the integrity of the lipid rafts, and thus on KinC activity. We constructed a double mutant $(\Delta f l o T \Delta y q f A)$, and used it to characterize the proteins associated with its DRM fraction. As shown in Supplemental Figure S10A, some proteins were missing in the DRM fraction of the $\Delta$ floT $\Delta y q f A$ double mutant. Western blot analyses also indicated a decrease in the KinC levels in this double mutant (Supplemental Fig. S10B). No effects were seen in either of the single mutants, suggesting that these two proteins have redundant function. We tested the KinC activity in the double mutant using an assay that depends on the ability of B. subtilis to form pellicles in LB medium in a KinCdependent manner when surfactin is added (Lopez et al. 2009). As shown in Figure 5, the $\Delta$ flot $\Delta y q f A$ double mutant phenocopies the $\Delta$ kinC mutant, both being unresponsive to surfactin. This indicates that in a cell lacking proteins with SPFH domains, KinC activity is indeed compromised. Interestingly, either overexpressing KinC or introducing the constitutively active allele of 


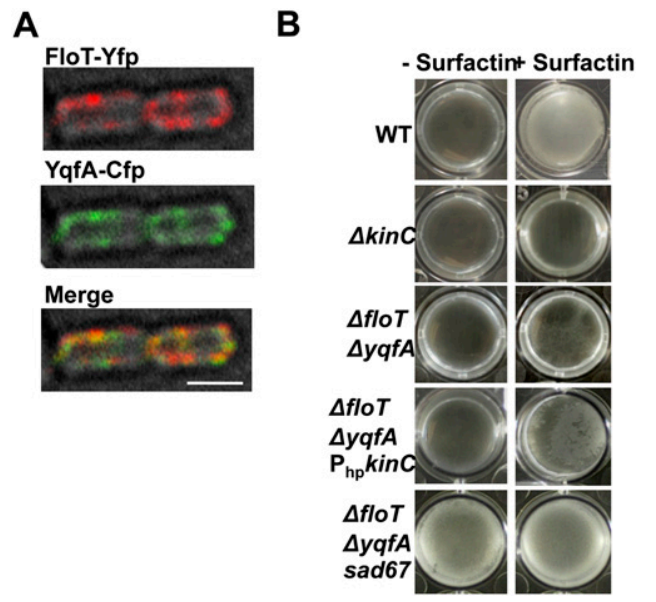

Figure 5. Colocalization of FloT and YqfA, the two SPFH domain proteins in $B$. subtilis. Deletion of floT and yqfA compromises KinC-dependent biofilm formation. (A) Colocalization of FloT and YqfA in a double-labeled strain expressing the translational fusions FloT-Yfp (false-colored in red) and YqfACfp (false-colored in green). Regions where the two signals overlapped appear yellow in the merge panel. Bar, $3 \mu \mathrm{m}(B)$ Deletion of floT and yqfA compromises KinC-dependent biofilm formation. The $\Delta f l o T \Delta y q f A$ double mutant was tested for its ability to form pellicles in response to the signaling molecule surfactin, when cultured in LB medium (Lopez et al. 2009). This behavior is dependent on the histidine kinase KinC, since the kinC-deficient mutant does not make pellicles when surfactin is added. The first and second rows of pictures show pellicles when surfactin was added to the wild-type strain, and the absence of pellicle formation in the kinC-deficient background, respectively. (Third row) A weak induction of pellicle formation was observed in the double mutant $\Delta f l o T \Delta y q f A$ when surfactin was added. (Fourth row) Overexpression of KinC in the double mutant $\Delta f l o T \Delta y q f A$ restored the ability to respond to surfactin by forming pellicles in the assay. (Fifth row) Introducing the allele sad67 in the double mutant $\Delta f l o T \Delta y q f A$ restored the ability to form pellicles.

spoOA sad67 in the $\Delta f l o T \Delta y q f A$ double mutant partially restored pellicle formation (Fig. 5B). A possible explanation for this partial restoration of activity would be that FloT might influence KinC activity by increasing local concentrations or promoting multimerization, similar to what has been observed in eukaryotic cells (Browman et al. 2007).

\section{Sequence homologs of Flotillin-1 from other bacteria also show punctate distribution}

Proteins showing sequence similarity to Flotillin-1 are widespread among bacteria. We thus wanted to test whether, in other bacterial species, these proteins also localize to discrete foci in the membrane. We constructed fusions of YFP to the Flotilin-1 sequence homologs SA1402 from Staphylococcus aureus and YqiK from E. coli. As shown in Figure 6A and Supplemental Figure S11, both of these fusion proteins displayed a punctate localization in the membrane. In $S$. aureus, the fusion protein localized to a single focus in the bacterial membrane. Consistent with the idea that this microdomain contained a lipid derived from squalene-probably staphyloxantin or a closely related molecule-localization of the protein was lost after treatment with zaragozic acid (Fig. 6A).

The above finding in $S$. aureus prompted us to analyze the protein content of the DRM fraction in this bacterium in a manner similar to what we did with $B$. subtilis (Supplemental Fig. S12). Proteins associated with the DRM fraction were sequenced, and, again, the majority of these proteins function in signal transduction, molecule trafficking, and protein secretion (Supplemental Table S1). Examples of the proteins present in the DRM fraction are the quorum-sensing regulator involved in virulence, CvfA (Nagata et al. 2008), and the elastinbinding protein EbpS, which is involved in biofilm formation and tissue colonization (Downer et al. 2002). A Flotillin-1 homolog (SA1402) and a KinC homolog (WalK) were also identified (Dubrac et al. 2007), as well the protease secretion machinery. This last finding is interesting, because there is a report suggesting that protease secretion in Gram (+) cocci may occur from a single location (Rosch and Caparon 2004). One possible interpretation is that such secretion may occur in the single domain observed in $S$. aureus. As can be observed in Figure 6B, treatment with zaragozic acid inhibited protease secretion, further supporting the hypothesis that this single location is related to protease secretion. Figure $6 \mathrm{~B}$ also shows a dramatic change in colony color as a consequence of zaragozic acid treatment. This is due to the fact that the yellow carotenoid staphyloxanthin from $S$. aureus is derived from squalene; thus, its synthesis is inhibited by zaragozic acid (Pelz et al. 2005).

Squalene synthesis inhibitors inhibit biofilm formation in B. subtilis and $\mathrm{S}$. aureus

Among the proteins that we found in the DRM fractions of both $B$. subtilis and S. aureus, several are involved in the process of biofilm formation. We thus wanted to

A

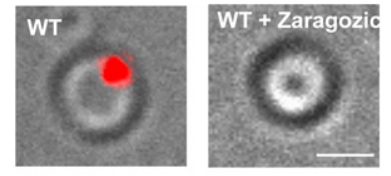

B

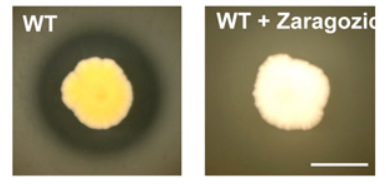

Figure 6. Lipid rafts in $S$. aureus. (A) Cellular localization of the translational fusion FloT-YFP in $S$. aureus (SA1402-YFP). The signal emitted is shown in red. Wild type untreated and treated with zaragozic acid are compared. Bar, $1 \mu \mathrm{m}$. (B) Inhibition of protease secretion in $S$. aureus using zaragozic acid. Secretion of proteases in casein-containing medium produces a clear halo due to the degradation of casein. The colony treated with zaragozic acid also shows inhibited production of the pigmented carotenoid staphyloxanthin. Bar, $1 \mathrm{~mm}$. 
assess the effect on biofilm formation of treating cultures with small molecule inhibitors that block various points along the pathway leading to squalene synthesis.

$B$. subtilis and $S$. aureus possess different routes to produce squalene (see Fig. 7A). S. aureus has the mevalonate route. This route is also present in humans, and can be inhibited by molecules-statins - that act on the key enzyme HMG-CoA reductase (Endo 1981; Wilding et al. 2000). In contrast, B. subtilis has the glyceraldehyde3-phosphate (GA3P) + pyruvate route (Takahashi et al. 1998). This route is also present in plants, and can be inhibited by clomazone, a strong inhibitor of 1-deoxy-Dxylulose-5-phosphate synthase (Mueller et al. 2000). Both routes converge into a single pathway with the formation of FPP. Thus, as shown above, inhibitors of squalene synthase, such as zaragozic acid, should have an effect on both $B$. subtilis and S. aureus. We tested these molecules

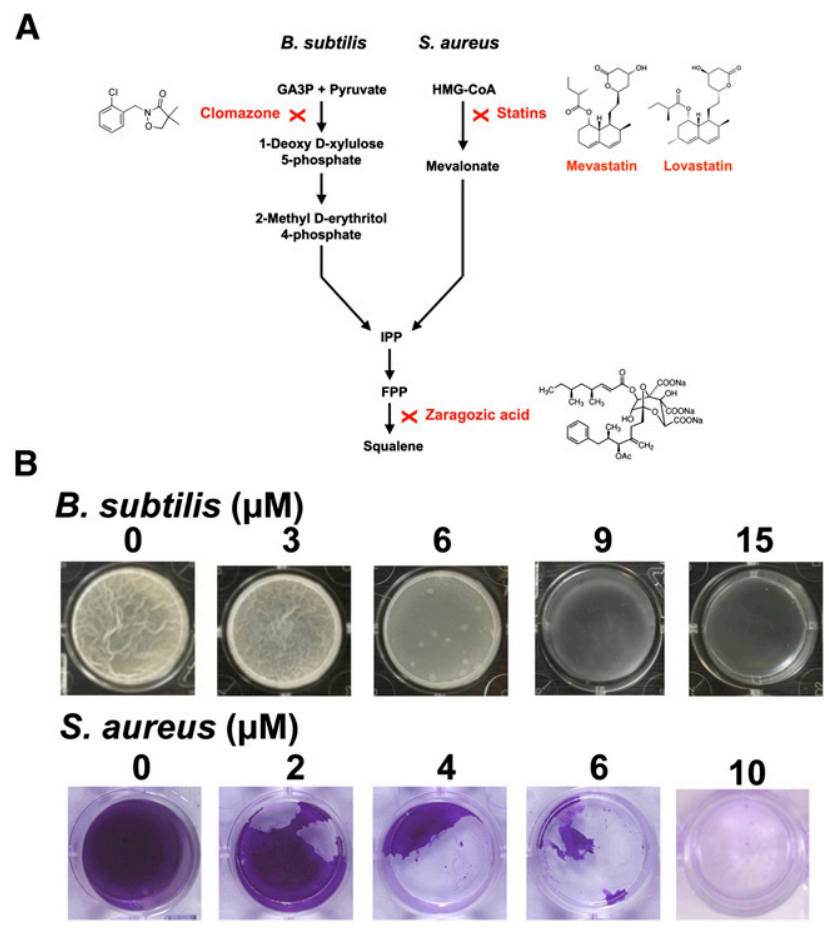

Figure 7. Inhibition of biofilm formation by sterol-lowering drugs. (A) Schematic representation of the two distinct biochemical pathways to produce squalene in $B$. subtilis and $S$. aureus. Zaragozic acid acts as a competitive inhibitor in both routes, since it acts downstream from the formation of FPP. Statins such as mevastin and lovastatin inhibit the enzyme HMG-CoA reductase, and thus the route to produce squalene in $S$. aureus. Clomazone inhibits the enzyme 1-deoxy-D-xylulose 5 -phosphate synthase, and thus the route to produce squalene in B. subtilis. (B) Addition of different concentrations of zaragozic acid to the biofilm formation assay of $B$. subtilis and $S$. aureus inhibited biofilm formation. Biofilm formation in B. subtilis is observed as a pellicle formed in the surface air-liquid of standing cultures, while $S$. aureus forms biofilms attached to the submerged surfaces (at the bottom of the well plate). Crystal violet staining was used in the $S$. aureus assay for better visualization. The effects of a range of drug concentration are shown. for their ability to affect biofilm formation in these two bacteria. The assays for each species are different due to the differences in the biofilms they make: $B$. subtilis forms floating pellicles that can be directly visualized, while $S$. aureus forms biofilms attached to submerged solid surfaces that are best visualized when stained (Fig. 7B; Supplemental Figs. S13, S14). Small concentrations of clomazone and zaragozic acid potently inhibited pellicle formation in B. subtilis; statins and zaragozic acid were potent biofilm inhibitors in $S$. aureus. However, both the statins and clomazone caused cell death due to the essential nature of several of the molecules made along these pathways. In contrast, zaragozic acid did not have a lethal effect. This feature might make zaragozic acid an attractive anti-biofilm agent, as one might expect a low incidence of resistance during the use of a nonlethal compound. More importantly, zaragozic acid treatment of $S$. aureus has the added advantage that both biofilm formation and virulence factors would be expected to be decreased simultaneously. Prior reports have indicated that other approaches at reducing biofilm formation in $S$. aureus lead to increased virulence and vice versa (Kong et al. 2006). In fact, aside from the biofilm inhibitory effects of squalene synthase inhibitors reported here, there is a report that squalene synthesis inhibitors inhibit S. aureus virulence (Liu et al. 2008).

\section{Discussion}

The finding that bacteria orchestrate several physiological processes in lipid rafts is exciting from several perspectives. Their conservation across the two most divergent domains of life argues strongly that these membrane microdomains are an ancient feature of cells. The ease of genetic, biochemical, and cell biological approaches in bacterial systems means that the functions of lipid rafts may be easier to study using a bacterial model system, which might contribute to clarify some controversial aspects inherent in the study of eukaryotic lipid rafts.

In relation to the function of lipid rafts in bacteria, we presume that the compartmentalization of specific proteins in tightly packed membrane areas might facilitate their activity. For example, the formation of protein complexes required for cell-cell communication (like the Opp signal-uptake machinery) or dimerization of membrane kinases (like KinC or WalK) —necessary in most cases to activate the cascades of signaling transduction-seems more feasible when these proteins are physically located in restricted membrane areas.

On a more practical note, it is possible that lipid rafts can be exploited as a new target to control bacterial infections. The fact that disrupting lipid rafts affects several key physiological processes associated with pathogenesis without killing the cell raises the possibility of "anti-raft" compounds as promising anti-infective agents. Remarkably, small molecules that inhibit raft formation simultaneously targeted diverse processes associated with infections in different bacteria; e.g., biofilm formation and exoprotease production. Given the enormous 
variability and complexity of these processes within bacterial species, and the difficulty in finding a standard treatment against them, the activity of the small molecules that we described in this study would be extremely helpful to combat chronic infections by simultaneously targeting many physiological processes associated to the infective process.

Interestingly, prior reports have indicated that other approaches to reducing biofilm formation lead to increased virulence and vice versa (Kong et al. 2006). As we showed above, the DRM of $S$. aureus harbor the proteins required for biofilm formation, attachment, virulence, and signaling. Thus, these processes may all be inhibited by blocking the formation of lipid rafts with the use of these small molecules. Supporting this finding, there is a study describing the use of inhibitors of the squalene synthesis to inhibit $S$. aureus virulence (Liu et al. 2008), in addition to our observations that squalene synthesis inhibitors also inhibit biofilm formation and exoprotease secretion. Furthermore, we observed that the use of zaragozic acid as an anti-raft drug does not affect bacterial growth. We postulate that, without any selective pressure caused by an agent that kills the cells, the use of zaragozic acid to inhibit bacterial infections might not give rise so rapidly to resistance mechanisms that are observed with many commonly used antibiotics.

In retrospect, there have been a number of studies describing the efficacy of statins to prevent infections. Patients treated to decrease high cholesterol levels have shown a remarkably reduced incidence of post-operative infection if previously treated with statins as cholesterollowering drugs. In other cases, previous treatment with statins strongly reduced the risk of hospitalization for sepsis in patients with chronic kidney disease who were receiving dialysis. Indeed, individuals receiving treatment with statins showed greatly reduced incidence of bacteremias caused by the pathogen $S$. aureus in hospitals (Liappis et al. 2001; Gupta et al. 2007; Falagas et al. 2008; Kopterides and Falagas 2009). In our opinion, it seems reasonable and promising that patients treated with these types of small molecules were protected against bacterial infections, as we postulated in this study. However, these studies are merely retrospective. More precise studies are now necessary to confirm this effect.

\section{Materials and methods}

\section{Strains, media, and culture conditions}

Strains used in this study were B. subtilis strain NCIB3610 (Branda et al. 2001) and S. aureus strains SC-1 and UAMS-1 (Beenken et al. 2003). Additional laboratory strains of E. coli DH5 $\alpha$, B. subtilis 168, and S. aureus RN4220 were required for cloning purposes. A list of strains used in this study is shown in Supplemental Table S3.

Biofilm formation assay for B. subtilis 3610 and S. aureus SC-1 and UAMS-1

Overnight cultures were diluted 1:100 in biofilm-inducing medium (MSgg for B. subtilis, and $3 \% \mathrm{TSB} \mathrm{NaCl}$ and $0.5 \%$ glucose for S. aureus SC-01), serum-coated surfaces were required for UAMS-1, as described previously (Beenken et al. 2003). Cells were dispensed in polystyrene well plates and incubated for $24 \mathrm{~h}$ at $30^{\circ} \mathrm{C}$ in the case of $B$. subtilis, or $37^{\circ} \mathrm{C}$ in the case of $S$. aureus. Biofilms formed by $S$. aureus were stained with crystal violet for better visualization, according to the protocol described by O'Toole and Kolter (1998). In S. aureus, biofilm formation was monitored quantitatively by dissolving the crystal violet associated with biofilms, and was measured spectrophotometrically at $\mathrm{OD}=595 \mathrm{~nm}$ (O'Toole and Kolter 1998). Exoprotease production assay was performed in LB plates supplemented with nonfat milk powder $(1 \%)$. Plates were incubated for $2 \mathrm{~d}$ at $37^{\circ} \mathrm{C}$. MSgg plates were supplemented with polyisopronoids for the $\Delta y i s P$ mutant complementation. Squalene, $\beta$-carotene, retinol, and staphyloxanthin were diluted into the medium at $100 \mu \mathrm{M}$ prior to the addition of cells. Pellicle formation in response to surfactin was assayed in LB medium. Strains were grown in LB shaking medium at $37^{\circ} \mathrm{C}$ in exponential phase for 20 generations. Cultures were transferred to polystyrene well plates, and surfactin was added to a final concentration of $20 \mu \mathrm{M}$. Cultures were incubated without shaking for $10 \mathrm{~h}$ at $30^{\circ} \mathrm{C}$ prior to imaging. Small molecules such as statins, clomazone, zaragozic acid, and surfactin were added directly to the pellicle formation assay at specified concentrations.

\section{Strain construction and reporters}

Deletion mutants $\Delta y i s P:: \mathrm{km}, \Delta s q \mathrm{hC}:: \mathrm{km}, \Delta s q \mathrm{hC}:: \mathrm{mls}$, $\Delta y u a G:: k m$, and $\Delta y q f A:: s p c$ were generated using long flanking homology PCR (using the primers listed in Supplemental Table S4; Wach 1996). For the translational fusion yuaG-yfp and for complementation of yis $P, a$ wild-type copy of yis $P$ was cloned in pKM008 or pKM003 vectors and inserted by double recombination into the neutral integration site $a m y E$ in the genome of $B$. subtilis strain 168 by inducing natural competence (Hardwood and Cutting 1990). Translational fusions yqiK-yfp and sa1402yfp were cloned in pKM008 and pMAD (Arnaud et al. 2004) plasmids, respectively, and were introduced into E. coli and $S$. aureus by electroporation. Colocalization of KinC with FloT in B. subtilis required overexpression of the translational fusion kinC-cfp under the control of the IPTG-inducible promoter $\mathrm{P}_{\text {hyperspank }}$ in $\mathrm{pDR} 111$. The construction $\mathrm{P}_{\text {hyperspank }}$-kinC-cfp was transferred to the plasmid pDR183 and inserted into the lacA neutral integration site by double recombination. Constructions were transferred to the strain NCIB3610 by phage transduction (Yasbin and Young 1974; Novick 1991).

\section{Image capture and analysis}

Colonies were photographed using a Zeiss Stemi SV6 stereoscope connected to a color AxioCam. Microscopy images were taken on a Nikon Eclipse TE2000-U microscope equipped with an X-cite 120 illumination system, using a Hamamatsu digital camera model ORCA-ER. Fluorescence signal was detected with a Ex436/500 filter. Image processing was done using MetaMorph software and Photoshop. Biofilm formation in well plates was photographed using a Nikon D100 digital camera.

\section{Overexpression and purification of YisP}

yis $P$ was expressed under the control of an IPTG-inducible promoter in the plasmid pET15-B (Novagen) using the restriction sites BamH1 and Ndel of the MCS. The protein expressed has an $\mathrm{N}$-terminal His-tagged sequence for further purification. Fivehundred milliliters of LB culture of E. coli BL21-DE3 Gold carrying the plasmid was grown in the presence of ampicillin to 
a density of $\mathrm{OD}_{600}=0.8$, and $0.5 \mathrm{mM}$ IPTG was added to induce expression. After $2 \mathrm{~h}$ of incubation, cells were pelleted and lysed using CellLytic B (Sigma). Purification of the His-tagged YisP was carried out using a His-select Nickel affinity gel (Sigma).

\section{Enzymatic activity and characterization of YisP}

An enzymatic assay to monitor the enzymatic activity of YisP was performed according to Debruyne (1983) with specific modifications. We monitored release of inorganic phosphate derived from the condensation of two molecules of FPP using the ability of rhodamine B to precipitate, once it is chelated with the inorganic phosphate released from the reaction of condensation $(2 \mathrm{FPP} \rightarrow$ Squalene $+2 \mathrm{PPi})$. The precipitate was washed, dissolved in acetone, and measured spectrophotometrically at 555 $\mathrm{nm}$. Two units of pyrophosphatase was added to the reaction mix to increase the sensitivity of the assay $(2 \mathrm{PPi} \rightarrow \mathrm{Pi}+\mathrm{Pi})$. The reaction buffer contained $50 \mathrm{mM}$ Tris- $\mathrm{HCl}$ and $20 \mathrm{mM} \mathrm{MgCl}_{2}(\mathrm{pH}$ 7.4), and different substrates (FPP, GPP, and GGPP) were used pairwise to optimize the reaction. Purified enzyme was preincubated in the reaction buffer for $20 \mathrm{~min}$ prior to enzymatic analysis.

Pigment production and analysis in $\mathrm{B}$. subtilis

To boost the production of pigment in B. subtilis, Msgg was mixed with $\mathrm{LB}$ (with $1 \% \mathrm{NaCl}$ ) at a ratio of $3: 1$, and $500 \mathrm{~mL}$ of culture was grown in a 1 - $\mathrm{L}$ flask shaking at $30^{\circ} \mathrm{C}$ until stationary phase was reached. Next, the culture was allowed to stand in the dark until color appeared $(\sim 8 \mathrm{~h})$. Cell pellets was dissolved in ethanol to $1: 1(\mathrm{v} / \mathrm{v})$ and incubated for $2 \mathrm{~h}$ at $45^{\circ} \mathrm{C}$. After centrifugation, the pellet was discarded and the ethanol containing the pigments was concentrated by evaporation $10 \times$ prior to LC-MS analysis.

\section{Cell membrane fractionation according} to detergent resistance

To purify the membrane fraction, cells were treated with lysozyme (B. subtilis) or lysostaphin (S. aureus) and lysed by sonication. Cell debris was eliminated by normal centrifugation $(13,000 \mathrm{rpm}$ for $2 \mathrm{~min})$, and the membrane fraction was precipitated from the supernatant by ultracentrifugation $(407,000 \mathrm{~g}$ for $40 \mathrm{~min})$. Next, the membrane fraction was treated with the CellLytic MEM protein extraction kit (Sigma) to purify proteins associated with DRM fractions. Separation of proteins associated with resistant and sensitive membrane fractions was done at $30^{\circ} \mathrm{C}$, according to the protocol provided by Sigma for the use of the CellLytic MEM protein extraction kit. Samples were run on SDS-PAGE, and proteins were detected by immunoblot blot, as described previously (Lopez et al. 2009), and by Coomasie staining. Coomasie-stained bands were analyzed by mass spectometry (Thermo Scientific LTQ Orbitrap XL).

\section{Acknowlegments}

We thank all members of the Kolter laboratory and the Losick laboratory for helpful discussions. We thank Chris Walsh, Rich Losick, and Michael Fischbach for helpful discussions. We thank the Chris Walsh laboratory (especially Michael Acker) and the John Clardy laboratory (especially Mohammad Seyedsayamdost) for their valuable help in characterizing the enzymology and the chemistry of YisP. We thank the Rudner laboratory (especially Professor David Rudner and Remi Bernard) for helpful discussion in strain construction and protein localization. This work was funded by a grant from the NIH to R.K. (GM58213). D.L. was the recipient of a post-doctoral fellowship from the Fundación Séneca, Comunidad Autónoma de la Región de Murcia (Spain).

\section{References}

Allen JA, Halverson-Tamboli RA, Rasenick MM. 2007. Lipid raft microdomains and neurotransmitter signalling. Nat Rev Neurosci 8: 128-140.

Arnaud M, Chastanet A, Debarbouille M. 2004. New vector for efficient allelic replacement in naturally nontransformable, low-GC-content, Gram-positive bacteria. Appl Environ Microbiol 70: 6887-6891.

Beenken KE, Blevins JS, Smeltzer MS. 2003. Mutation of sarA in Staphylococcus aureus limits biofilm formation. Infect Immun 71: 4206-4211.

Bergstrom JD, Kurtz MM, Rew DJ, Amend AM, Karkas JD, Bostedor RG, Bansal VS, Dufresne C, VanMiddlesworth FL, Hensens OD, et al. 1993. Zaragozic acids: A family of fungal metabolites that are picomolar competitive inhibitors of squalene synthase. Proc Natl Acad Sci 90: 80-84.

Bolard J. 1986. How do the polyene macrolide antibiotics affect the cellular membrane properties? Biochim Biophys Acta 864: 257-304.

Bosak T, Losick RM, Pearson A. 2008. A polycyclic terpenoid that alleviates oxidative stress. Proc Natl Acad Sci 105: 6725-6729.

Branda SS, Gonzalez-Pastor JE, Ben-Yehuda S, Losick R, Kolter R. 2001. Fruiting body formation by Bacillus subtilis. Proc Natl Acad Sci 98: 11621-11626.

Branda SS, Gonzalez-Pastor JE, Dervyn E, Ehrlich SD, Losick R, Kolter R. 2004. Genes involved in formation of structured multicellular communities by Bacillus subtilis. I Bacteriol 186: 3970-3979.

Branda SS, Chu F, Kearns DB, Losick R, Kolter R. 2006. A major protein component of the Bacillus subtilis biofilm matrix. Mol Microbiol 59: 1229-1238.

Browman DT, Hoegg MB, Robbins SM. 2007. The SPFH domaincontaining proteins: More than lipid raft markers. Trends Cell Biol 17: 394-402.

Brown DA. 2002. Isolation and use of rafts. Curr Protoc Immunol 51: 11.10.1-11.10.23.

Butcher BG, Helmann JD. 2006. Identification of Bacillus subtilis $\sigma$-dependent genes that provide intrinsic resistance to antimicrobial compounds produced by Bacilli. Mol Microbiol 60: 765-782.

Debruyne I. 1983. Inorganic phosphate determination: Colorimetric assay based on the formation of a rhodamine B-phosphomolybdate complex. Anal Biochem 130: 454-460.

Donovan C, Bramkamp M. 2009. Characterization and subcellular localization of a bacterial flotillin homologue. Microbiology 155: 1786-1799.

Dorenbos R, Stein T, Kabel J, Bruand C, Bolhuis A, Bron S, Quax WJ, Van Dijl JM. 2002. Thiol-disulfide oxidoreductases are essential for the production of the lantibiotic sublancin 168 . J Biol Chem 277: 16682-16688.

Downer R, Roche F, Park PW, Mecham RP, Foster TJ. 2002. The elastin-binding protein of Staphylococcus aureus (EbpS) is expressed at the cell surface as an integral membrane protein and not as a cell wall-associated protein. I Biol Chem 277: 243-250.

Dubrac S, Boneca IG, Poupel O, Msadek T. 2007. New insights into the WalK/WalR (YycG/YycF) essential signal transduction pathway reveal a major role in controlling cell wall metabolism and biofilm formation in Staphylococcus aureus. J Bacteriol 189: 8257-8269.

Endo A. 1981. 3-Hydroxy-3-methylglutaryl-CoA reductase inhibitors. Methods Enzymol 72: 684-689.

Falagas ME, Makris GC, Matthaiou DK, Rafailidis PI. 2008. Statins for infection and sepsis: A systematic review of the clinical evidence. I Antimicrob Chemother 61: 774-785. 
Gupta R, Plantinga LC, Fink NE, Melamed ML, Coresh J, Fox CS, Levin NW, Powe NR. 2007. Statin use and sepsis events [corrected] in patients with chronic kidney disease. IAMA 297: 1455-1464.

Hardwood CR, Cutting SM. 1990. Molecular biological methods for Bacillus. Wiley, New York.

Hinderhofer M, Walker CA, Friemel A, Stuermer CA, Moller HM, Reuter A. 2009. Evolution of prokaryotic SPFH proteins. BMC Evol Biol 9: 10. doi: 10-1186/1471-2148-9-10.

Ireton K, Rudner DZ, Siranosian KJ, Grossman AD. 1993. Integration of multiple developmental signals in Bacillus subtilis through the Spo0A transcription factor. Genes Dev 7: 283-294.

Kato N, Nakanishi M, Hirashima N. 2006. Flotillin-1 regulates IgE receptor-mediated signaling in rat basophilic leukemia (RBL-2H3) cells. I Immunol 177: 147-154.

Kawai F, Shoda M, Harashima R, Sadaie Y, Hara H, Matsumoto K. 2004. Cardiolipin domains in Bacillus subtilis marburg membranes. J Bacteriol 186: 1475-1483.

Kobayashi K, Ehrlich SD, Albertini A, Amati G, Andersen KK, Arnaud M, Asai K, Ashikaga S, Aymerich S, Bessieres P, et al. 2003. Essential Bacillus subtilis genes. Proc Natl Acad Sci 100: 4678-4683.

Kong KF, Vuong C, Otto M. 2006. Staphylococcus quorum sensing in biofilm formation and infection. Int J Med Microbiol 296: 133-139.

Kontinen VP, Sarvas M. 1993. The PrsA lipoprotein is essential for protein secretion in Bacillus subtilis and sets a limit for high-level secretion. Mol Microbiol 8: 727-737.

Kontnik R, Bosak T, Butcher RA, Brocks JJ, Losick R, Clardy J, Pearson A. 2008. Sporulenes, heptaprenyl metabolites from Bacillus subtilis spores. Org Lett 10: 3551-3554.

Kopterides P, Falagas ME. 2009. Statins for sepsis: A critical and updated review. Clin Microbiol Infect 15: 325-334.

Langhorst MF, Reuter A, Stuermer CA. 2005. Scaffolding microdomains and beyond: The function of reggie/flotillin proteins. Cell Mol Life Sci 62: 2228-2240.

Lazazzera BA, Solomon JM, Grossman AD. 1997. An exported peptide functions intracellularly to contribute to cell density signaling in B. subtilis. Cell 89: 917-925.

Lee S, Poulter CD. 2008. Cloning, solubilization, and characterization of squalene synthase from Thermosynechococcus elongatus BP-1. J Bacteriol 190: 3808-3816.

Letunic I, Doerks T, Bork P. 2009. SMART 6: Recent updates and new developments. Nucleic Acids Res 37: D229-D232. doi: 10.1093/nar/gkn808.

Liappis AP, Kan VL, Rochester CG, Simon GL. 2001. The effect of statins on mortality in patients with bacteremia. Clin Infect Dis 33: 1352-1357.

Lingwood D, Simons K. 2010. Lipid rafts as a membraneorganizing principle. Science 327: 46-50.

Liu CI, Liu GY, Song Y, Yin F, Hensler ME, Jeng WY, Nizet V, Wang AH, Oldfield E. 2008. A cholesterol biosynthesis inhibitor blocks Staphylococcus aureus virulence. Science 319: 1391-1394.

Lopez D, Fischbach MA, Chu F, Losick R, Kolter R. 2009. Structurally diverse natural products that cause potassium leakage trigger multicellularity in Bacillus subtilis. Proc Natl Acad Sci 106: 280-285.

Matsumoto K, Kusaka J, Nishibori A, Hara H. 2006. Lipid domains in bacterial membranes. Mol Microbiol 61: 1110-1117.

Meile JC, Wu LJ, Ehrlich SD, Errington J, Noirot P. 2006. Systematic localisation of proteins fused to the green fluorescent protein in Bacillus subtilis: Identification of new proteins at the DNA replication factory. Proteomics 6: 21352146.
Michel V, Bakovic M. 2007. Lipid rafts in health and disease. Biol Cell 99: 129-140.

Mileykovskaya E, Dowhan W. 2009. Cardiolipin membrane domains in prokaryotes and eukaryotes. Biochim Biophys Acta 1781: 2084-2091.

Morrow IC, Parton RG. 2005. Flotillins and the PHB domain protein family: Rafts, worms and anaesthetics. Traffic 6: $725-740$.

Mueller C, Schwender J, Zeidler J, Lichtenthaler HK. 2000. Properties and inhibition of the first two enzymes of the nonmevalonate pathway of isoprenoid biosynthesis. Biochem Soc Trans 28: 792-793.

Nagata M, Kaito C, Sekimizu K. 2008. Phosphodiesterase activity of CvfA is required for virulence in Staphylococcus aureus. J Biol Chem 283: 2176-2184.

Novick RP. 1991. Genetic systems in staphylococci. Methods Enzymol 204: 587-636.

O'Toole GA, Kolter R. 1998. Initiation of biofilm formation in Pseudomonas fluorescens WCS365 proceeds via multiple, convergent signalling pathways: A genetic analysis. Mol Microbiol 28: 449-461.

Ollinger J, Song KB, Antelmann H, Hecker M, Helmann JD. 2006. Role of the Fur regulon in iron transport in Bacillus subtilis. J Bacteriol 188: 3664-3673.

Pelz A, Wieland KP, Putzbach K, Hentschel P, Albert K, Gotz F. 2005. Structure and biosynthesis of staphyloxanthin from Staphylococcus aureus. I Biol Chem 280: 32493-32498.

Pike LJ. 2006. Rafts defined: A report on the Keystone symposium on lipid rafts and Ccell function. I Lipid Res 47: 15971598.

Romero D, Aguilar C, Losick R, Kolter R. 2010. Amyloid fibers provide structural integrity to Bacillus subtilis biofilms. Proc Natl Acad Sci 107: 2230-2234.

Rosch J, Caparon M. 2004. A microdomain for protein secretion in Gram-positive bacteria. Science 304: 1513-1515.

Schultz J, Milpetz F, Bork P, Ponting CP. 1998. SMART, a simple modular architecture research tool: Identification of signaling domains. Proc Natl Acad Sci 95: 5857-5864.

Takahashi S, Kuzuyama T, Watanabe H, Seto H. 1998. A 1-deoxy-D-xylulose 5-phosphate reductoisomerase catalyzing the formation of 2-C-methyl-D-erythritol 4-phosphate in an alternative nonmevalonate pathway for terpenoid biosynthesis. Proc Natl Acad Sci 95: 9879-9884.

Tavernarakis N, Driscoll M, Kyrpides NC. 1999. The SPFH domain: Implicated in regulating targeted protein turnover in stomatins and other membrane-associated proteins. Trends Biochem Sci 24: 425-427.

Wach A. 1996. PCR-synthesis of marker cassettes with long flanking homology regions for gene disruptions in S. cerevisiae. Yeast 12: 259-265.

Watanabe M, Wake H, Moorhouse AJ, Nabekura J. 2009. Clustering of neuronal $\mathrm{K}^{+}-\mathrm{Cl}^{-}$cotransporters in lipid rafts by tyrosine phosphorylation. J Biol Chem 284: 27980-27988.

Wilding EI, Brown JR, Bryant AP, Chalker AF, Holmes DJ, Ingraham KA, Iordanescu S, So CY, Rosenberg M, Gwynn MN. 2000. Identification, evolution, and essentiality of the mevalonate pathway for isopentenyl diphosphate biosynthesis in gram-positive cocci. J Bacteriol 182: 4319-4327.

Yasbin RE, Young FE. 1974. Transduction in Bacillus subtilis by bacteriophage SPP1. J Virol 14: 1343-1348.

Zhang HM, Li Z, Tsudome M, Ito S, Takami H, Horikoshi K. 2005. An alkali-inducible flotillin-like protein from Bacillus halodurans C-125. Protein I 24: 125-131. 


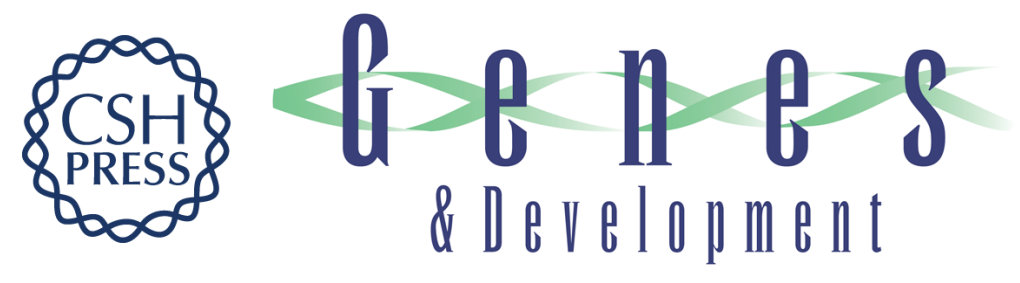

\section{Functional microdomains in bacterial membranes}

Daniel López and Roberto Kolter

Genes Dev. 2010, 24: originally published online August 16, 2010

Access the most recent version at doi:10.1101/gad.1945010

Supplemental
Material http://genesdev.cshlp.org/content/suppl/2010/08/10/gad.1945010.DC1

References This article cites 59 articles, 28 of which can be accessed free at:

http://genesdev.cshlp.org/content/24/17/1893.full.html\#ref-list-1

License

Email Alerting Receive free email alerts when new articles cite this article - sign up in the box at the top Service right corner of the article or click here.

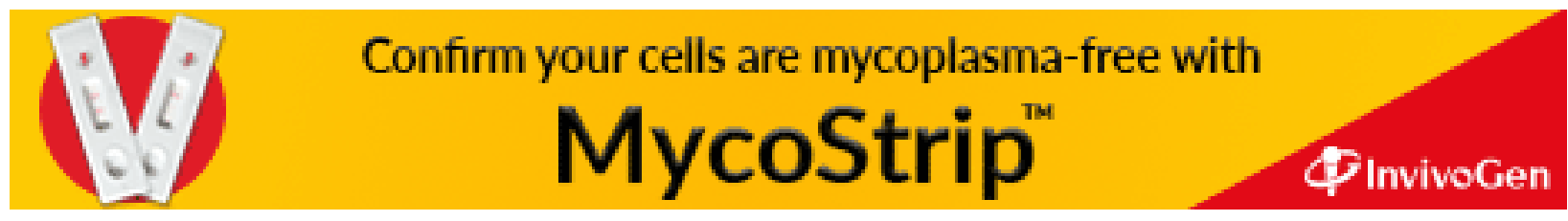

